\title{
NEAT-FLAT MODULES
}

\section{Engin Büyükaşık ${ }^{1}$ and Yılmaz Durğun ${ }^{2}$}

${ }^{1}$ Department of Mathematics, Izmir Institute of Technology, Urla, Izmir, Turkey

${ }^{2}$ Department of Mathematics, Bitlis Eren University, Bitlis, Turkey

Let $R$ be a ring. A right $R$-module $M$ is said to be neat-flat if the kernel of any epimorphism $Y \rightarrow M$ is neat in $Y$, i.e., the induced map $\operatorname{Hom}(S, Y) \rightarrow \operatorname{Hom}(S, M)$ is surjective for any simple right $R$-module $S$. Neat-flat right $R$-modules are projective if and only if $R$ is a right $\sum-C S$ ring. Every cyclic neat-flat right $R$-module is projective if and only if $R$ is right $C S$ and right $C$-ring. It is shown that, over a commutative Noetherian ring $R$, (1) every neat-flat module is flat if and only if every absolutely coneat module is injective if and only if $R \cong A \times B$, wherein $A$ is a $Q F$-ring and $B$ is hereditary, and (2) every neat-flat module is absolutely coneat if and only if every absolutely coneat module is neat-flat if and only if $R \cong A \times B$, wherein $A$ is a $Q F$-ring and $B$ is Artinian with $J^{2}(B)=0$.

Key Words: Closed submodule; (Co)neat submodule; Extending module; Neat-flat module; $Q F$-ring.

2010 Mathematics Subject Classification: 16D10; 16D40; $16 \mathrm{E} 30$.

\section{INTRODUCTION}

Throughout, $R$ is an associative ring with identity and all modules are unitary right $R$-modules. For an R-module $M, E(M), \operatorname{Soc}(M)$ will denote the injective hull, the socle of $M$, respectively. The character module $\operatorname{Hom}_{Z}(M, \mathbb{Q} / \mathbb{Z})$ of $M$ is denoted by $M^{+}$. The Jacobson radical of the $\operatorname{ring} R$ is denoted by $J(R)$.

A submodule $K$ of an $R$-module $M$ is called closed (in $M$ ) provided $K$ has no proper essential extension in $M$. When $R$ is a Dedekind domain (more generally a Prüfer domain), a submodule $K$ of an R-module $M$ is said to be pure if and only if $K \cap a M=a K$ for all $a \in R$. Inspired by this characterization of pure submodules over Dedekind domains, Honda [14] introduced neat subgroups in order to characterize closed subgroups in abelian groups. Namely, a subgroup $A$ of an abelian group $B$ is called neat in $B$ if $A p=A \cap B p$ for every prime $p$. A subgroup $A$ of an abelian group $B$ is closed if and only if it is neat if and only if $\operatorname{Hom}(S, B) \rightarrow \operatorname{Hom}(S, B / A) \rightarrow 0$ is surjective for each simple $R$-module $S$. Neatness over arbitrary associative rings considered by Renault [20], namely, a submodule $A$ of an $R$-module $B$ is called neat if $\operatorname{Hom}(S, B) \rightarrow \operatorname{Hom}(S, B / A) \rightarrow 0$ is surjective

Received August 14, 2014; Revised October 3, 2014; Communicated by E. Puczylowski.

Address correspondence to Dr. Yllmaz Durğun, Bitlis Eren University, Department of Mathematics, Bitlis, Turkey; E-mail: ydurgun@beu.edu.tr 
for each simple $R$-module $S$. Closed submodules are neat, but the converse is true exactly for $C$-rings (i.e., $\operatorname{Soc}(R / I) \neq 0$ for every proper essential right ideal $I$ of $R$ ).

A submodule $K$ of $M$ is called small in $M$ if $M \neq K+T$ for every proper submodule $T$ of $M$. As the dual of closed submodule, the submodule $K$ is called coclosed in $M$ if for every submodule $A$ of $M$ with $A \leq K, K / A \ll M / A$ implies $K=$ $A$. Recently, Zöschinger showed in [28] that, over a commutative Noetherian ring $R$, closed submodules are coclosed if and only if coclosed submodules are closed if and only if $R$ is distributive. In his recent article, as a dual of neat submodule, Fuchs [10] defined a submodule $N$ of $M$ to be coneat if $\operatorname{Hom}(M, S) \rightarrow \operatorname{Hom}(N, S) \rightarrow 0$ is surjective for each simple $R$-module $S$. In that article, he proved that for an integral domain $R$ neat submodules and coneat submodules coincide if and only if every maximal ideal of $R$ is finitely generated. Crivei is also concerned with the same problem in [7], and he showed that if $R$ is a commutative ring whose maximal ideals are principal then neat and coneat submodules of every module coincide.

Recently, there is a significant interest to some classes of modules that are defined via (co) closed submodules and (co) neat submodules, (see, [7, 17, 2528]). An $R$-module $M$ is said to be $m$-injective (weakly-injective, absolutely coneat, respectively) if it is neat (coclosed, coneat, respectively) in every extension. Note that closed submodule of an injective module is injective. $m$-injective modules are injective if and only if every neat submodule is closed (i.e., $R$ is a right $C$-ring), (see [24]). Weakly-injective modules are introduced and discussed by Zöschinger in [27, 28]). Absolutely coneat modules are introduced and studied by Crivei in [7].

Motivating by the relation between weakly-flat modules and closed submodules, we investigate the modules $M$, for which any short exact sequence ending with $M$ is neat-exact. Namely, we say $M$ is neat-flat if the kernel of any epimorphism $Y \rightarrow M$ is neat in $Y$, i.e., the induced map $\operatorname{Hom}(S, Y) \rightarrow \operatorname{Hom}(S, M)$ is surjective for any simple $R$-module $S$. Projective modules, weakly-flat modules, and nonsingular modules are neat-flat. In [17], the author introduced simpleprojective modules to characterize the rings whose simple modules have projective (pre)envelope. An $R$-module $M$ is called simple-projective if for any simple right $R$-module $N$, every homomorphism $f: N \rightarrow M$ factors through a finitely generated free right $R$-module $F$.

The article is organized as follows. In Section 2, it is shown that neat-flat modules coincide with simple-projective modules over arbitrary rings. Next, we give the main properties of the class of neat-flat $R$-modules. The right socle of $R$ is zero if and only if neat-flat modules coincide with the modules that have zero socle. A ring $R$ is a right $C$-ring if and only if neat-flat modules are weakly-flat. We also investigate the rings over which neat-flat modules are projective. Namely, we prove that, (1) every neat-flat module is projective if and only if $R$ is a right $\sum-C S$ ring; (2) every finitely generated neat-flat module is projective if and only if $R$ is a right $C$-ring and every finitely generated free right $R$-module is extending; and (3) every cyclic right $R$-module is projective if and only if $R$ is right $C S$ and right $C$-ring.

In Section 3, it is shown that, over a commutative Noetherian ring $R,(1)$ every neat-flat module is flat if and only if every absolutely coneat module is injective if and only if $R \cong A \times B$, wherein $A$ is $Q F$-ring and $B$ is hereditary; and (2) every neat-flat module is absolutely coneat if and only if every absolutely coneat module is neat-flat if and only if every neat-flat module is weakly-injective if and only if 
every absolutely coneat module is weakly-flat if and only if $R \cong A \times B$, wherein $A$ is $Q F$-ring and $B$ is Artinian with $J^{2}(B)=0$.

In Section 4, localization of neat exact sequences and neat-flat modules are investigated. It is shown that, over a commutative $N$-ring $R$, (1) a short exact sequence $0 \rightarrow A \rightarrow B \rightarrow C \rightarrow 0$ is neat exact, i.e., $A$ is neat in $B$ if and only if $0 \rightarrow$ $A_{P} \rightarrow B_{P} \rightarrow C_{P} \rightarrow 0$ is neat exact for each maximal ideal $P$ of $R$; and (2) a module $M$ is neat-flat if and only if, for all maximal ideals $P$ of $R, M_{P}$ is neat-flat $R_{P}$-module.

For the unexplained concepts and results, we refer the reader to $[1,4]$ and [16].

\section{NEAT-FLAT MODULES}

Let $\mathbb{E}: 0 \rightarrow K \stackrel{f}{\rightarrow} L \stackrel{g}{\rightarrow} M \rightarrow 0$ be a short exact sequence. $\mathbb{E}$ is called neat exact if $f(K)$ is a neat submodule of $L$. In this case, $f$ and $g$ are called neat monomorphism and neat epimorphism, respectively. By definition, the class of neat exact sequences is projectively generated by the class of simple $R$-modules. Hence neat-exact sequences form a proper class in the sense of Bushbaum, (see[4, 10.8]). For the following lemma we refer to [18, Proposition 1.12-1.13]. The proof is included for completeness.

Lemma 2.1. The following statements are equivalent for a right $R$-module $M$ :

(1) $M$ is neat-flat;

(2) Every exact sequence $0 \rightarrow A \rightarrow B \rightarrow M \rightarrow 0$ is neat exact;

(3) There exists a neat exact sequence $0 \rightarrow K \rightarrow F \rightarrow M \rightarrow 0$ with $F$ projective;

(4) There exists a neat exact sequence $0 \rightarrow K \rightarrow F \rightarrow M \rightarrow 0$ with $F$ neat-flat.

Proof. $\quad(1) \Rightarrow(2) \Rightarrow(3) \Rightarrow(4)$ are clear.

(4) $\Rightarrow$ (1) Let $0 \rightarrow A \rightarrow B \stackrel{g}{\rightarrow} M \rightarrow 0$ be any short exact sequence. We claim that $g$ is a neat epimorphism, i.e., $\operatorname{Ker}(g)$ is a neat submodule of $B$. By (4), there exists a neat exact sequence $0 \rightarrow K \stackrel{f}{\rightarrow} F \stackrel{s}{\rightarrow} M \rightarrow 0$ with $F$ neat-flat. Considering the pullback of $g$ and $s$, we obtain a commutative diagram with exact rows

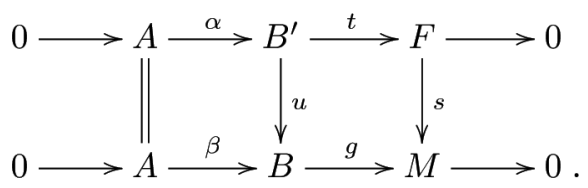

Since $F$ is neat-flat, $\alpha(A)$ is neat in $B^{\prime}$. As $\alpha(A)$ is neat in $B^{\prime}$ and $f(K)$ is neat in $F$, we have $\beta(A)$ is neat in $B$ by $[4,10.1]$. This completes the proof.

\section{Remark 2.2.}

(1) Clearly, if $\operatorname{Soc}(M)$ is projective, then $M$ is neat-flat. In particular, if $M$ has no simple submodules, then $M$ is neat-flat.

(2) Obviously, projective modules are neat-flat. On the other hand, the infinite direct product of the ring of integers $\mathbb{Z}$ is neat-flat, but not projective.

(3) Note that a simple right $R$-module is neat-flat if and only if it is projective. Thus $R$ is a semisimple Artinian ring if and only if every right R-module is neat-flat. 
(4) By [22, Lemma 2.3(a)], every nonsingular module is weakly-flat. Since weaklyflat modules are neat-flat, nonsingular modules are neat-flat. modules.

The following observation is useful for the further characterization of neat-flat

Lemma 2.3. Let $R$ be a ring. An R-module $M$ is simple-projective if and only if $M$ is neat-flat.

Proof. Suppose $M$ is simple-projective and $s: R^{(I)} \rightarrow M$ be an epimorphism. Let $S$ be simple right $R$-module and $f: S \rightarrow M$ be a homomorphism. As $M$ is simple-projective $f$ factors through a finitely generated free module, i.e., there are homomorphisms $h: S \rightarrow R^{n}$ and $g: R^{n} \rightarrow M$ such that $f=g h$. Since $R^{n}$ is projective, there is a homomorphism $t: R^{n} \rightarrow R^{(I)}$ such that $g=$ st. We get the following diagram:

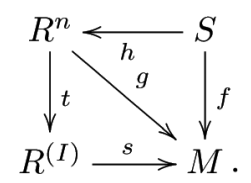

Then $f=g h=s t h$, and so the induced map $\operatorname{Hom}\left(S, R^{(I)}\right) \rightarrow \operatorname{Hom}(S, M) \rightarrow 0$ is surjective. Therefore, the sequence $0 \rightarrow \operatorname{Ker} s \rightarrow R^{(I)} \stackrel{s}{\rightarrow} M \rightarrow 0$ is neat exact. Hence $M$ is neat-flat by Lemma 2.1(3).

Conversely, let $M$ be a neat-flat module. Then there is a neat exact sequence $0 \rightarrow K \rightarrow F \stackrel{g}{\rightarrow} M \rightarrow 0$ with $F$ free by Lemma 2.1 . Let $S$ be a simple module and $f: S \rightarrow M$ be any homomorphism. Then there is a homomorphism $h: S \rightarrow F$ such that $f=g h$. As $S$ is finitely generated, $h(S) \leq H$ for some finitely generated free submodule of $F$. Then we get $f=g h=(g i) h^{\prime}$, where $i: H \rightarrow F$ is the inclusion and $h^{\prime}: S \rightarrow H$ is the homomorphism defined as $h^{\prime}(x)=h(x)$ for each $x \in S$. Therefore, $f$ factors through $H$, and so $M$ is simple projective.

From the proof of the lemma above, we have the following corollary.

Corollary 2.4. If $M$ is a neat-flat right $R$-module, then any simple submodule of $M$ is isomorphic to a minimal right ideal of $R$.

Let $M$ be a module with $\operatorname{Soc}(M)=0$. Then $\operatorname{Hom}(S, M)=0$ for any simple right $R$-module $S$, and so $M$ is neat-flat. Corollary 2.4 yields the following corollary.

Corollary 2.5. Let $R$ be a ring. The following statements are equivalent:

(1) $\operatorname{Soc}\left(R_{R}\right)=0$;

(2) An R-module $M$ is neat-flat if and only if $\operatorname{Soc}\left(M_{R}\right)=0$.

Proposition 2.6. The class of neat-flat R-modules is closed under extensions, direct sums, pure submodules, and direct summands.

Proof. By Lemma 2.3 and [17, Proposition 2.4]. 
Recall that a ring $R$ is called a right $C$-ring if $\operatorname{Soc}(M) \neq 0$ for every (cyclic) singular $R$-module $M$. Left perfect rings, right semiartinian rings and almost perfect domains are right $C$-rings. $R$ is a right $C$-ring if and only if neat submodules are closed if and only if $m$-injective modules are injective, (see [24, Lemma 4], [11, Theorem 5]).

Following, Zöschinger [28], a right $R$-module $M$ is called weakly-flat if the kernel of any epimorphism $Y \rightarrow M \rightarrow 0$ is a closed submodule of $Y$. Every nonsingular module is weakly-flat, and the converse is true exactly when the underlying ring is nonsingular (see, [22, Lemma 2.3]).

Proposition 2.7. A ring $R$ is a right $C$-ring if and only if neat-flat are $R$-modules are weakly-flat.

Proof. Necessity is clear. For the sufficiency suppose an $R$-module $M$ is $m$-injective. We claim that $M$ is injective. Consider the exact sequence $0 \rightarrow M \hookrightarrow E(M) \rightarrow$ $E(M) / M \rightarrow 0$. By [6, Theorem 3], $\operatorname{Soc}(E(M) / M)=0$, and so $E(M) / M$ is neat-flat. Now, $M$ is closed in $E(M)$ by the hypothesis. Therefore, $M$ is injective, so $R$ is a right $C$-ring by [24, Lemma 4].

Corollary 2.8. A ring $R$ is right $C$-ring and right nonsingular if and only if neat-flat modules are nonsingular.

The following result is a generalization of [28, Satz 1.1].

Proposition 2.9. Let $R$ be a right $C$-ring and $M$ be a right $R$-module. The following statements are equivalent:

(1) $M$ is weakly-flat;

(2) $M$ is neat-flat;

(3) $\operatorname{Soc}(M)=M \cdot \operatorname{Soc}\left(R_{R}\right)$.

Proof. $\quad(1) \Leftrightarrow(2)$ By Proposition 2.7 .

(2) $\Rightarrow$ (3) Let $S$ be simple submodule of $M$. Then the inclusion map $i: S \rightarrow$ $M$ factors through $R$ by Lemma 2.3. That is, there are homomorphisms $f: S \rightarrow R$ and $g: R \rightarrow M$ such that $g f=i$. As $S$ is simple, $f(S)=A_{R}$ is a simple right ideal of $R$. Therefore $S=i(S)=g f(S)=g(A)=g(R) A \leq M \cdot \operatorname{Soc}\left(R_{R}\right)$. Hence $\operatorname{Soc}(M) \leq$ $M . \operatorname{Soc}\left(R_{R}\right)$. The reverse containment is clear.

(3) $\Rightarrow$ (2) Suppose $M \cong F / K$ for some free module $F$ and a submodule $K$ of $F$. Assume $K$ is not closed in $F$. Then there is a submodule $T$ of $F$ containing $K$ essentially. Now $\operatorname{Soc}(T / K) \neq 0$, because $T / K$ is singular and $R$ is right $C$-ring. Let $A$ be a complement of $K$ in $F$. Then $A \oplus K$ is essential in $F$, and $\operatorname{so} \operatorname{Soc}(F)=$ $\operatorname{Soc}(A) \oplus \operatorname{Soc}(K)$. We get $\operatorname{Soc}\left(\frac{F}{K}\right)=\left(\frac{F}{K}\right) \operatorname{Soc}\left(R_{R}\right)=\frac{(\operatorname{Soc}(F)+K)}{K}=\frac{(\operatorname{Soc}(A)+K)}{K}$. Therefore $\frac{T}{K} \cap\left[\frac{(\operatorname{Soc}(A)+K)}{K}\right] \neq 0$, and this implies $A \cap K \neq 0$, a contradiction. Hence $K$ is a closed submodule of $F$, and so $M$ is weakly-flat.

A module $M$ is said to be extending or a $C S$-module if every closed submodule of $M$ is a direct summand of $M . R$ is a right $C S$ ring if $R_{R}$ is $C S . M$ is called $\sum-C S$ module if every direct sum of copies of $M$ is $C S$, (see [8]). The $\sum-C S$ rings were first introduced and termed as co- $H$-rings in [19]. 
Theorem 2.10. Let $R$ be a ring. The following statements are equivalent:

(1) Every neat-flat $R$-module is projective;

(2) $R$ is a right $\sum-C S$ ring.

Proof. $\quad(1) \Rightarrow(2)$ Let $P$ be a projective $R$-module and $N$ be a closed submodule of $P$. Then $P / N$ is neat-flat by Lemma 2.1 , and so $P / N$ is projective by (1). Therefore, the sequence $0 \rightarrow N \rightarrow P \rightarrow P / N \rightarrow 0$ splits, and so $N$ is a direct summand of $P$. Hence $R$ is a $\sum-C S$ ring.

$(2) \Rightarrow(1)$ Every right $\sum-C S$ ring is both right and left perfect by [19, Theorem 3.18]. Hence, $R$ is a right $C$-ring by [1, Theorem 28.4]. Let $M$ be a neat-flat $R$-module. Then there is a neat exact sequence $0 \rightarrow K \hookrightarrow P \rightarrow M \rightarrow 0$ with $P$ projective by Lemma 2.1. Since $R$ is a right $C$-ring, $K$ is closed in $P$ by [11, Theorem 5]. By the assumption, $K$ is direct summand in $P$, and so $M$ is projective.

Theorem 2.11. Let $R$ be a ring. The following statements are equivalent:

(1) Every finitely generated neat-flat $R$-module is projective;

(2) $R$ is a right $C$-ring and every finitely generated free $R$-module is extending.

Proof. $\quad(1) \Rightarrow(2)$ Let $I$ be an essential right ideal of $R$ with $\operatorname{Soc}(R / I)=0$. Then $\operatorname{Hom}(S, R / I)=0$ for each simple $R$-module $S$, and hence $I$ is neat ideal of $R$. So $R / I$ is neat-flat by Lemma 2.1. But it is projective by (1), and so $I$ is direct summand of $R$. This contradicts with essentiality of $I$ in $R$. So that $R$ is a right $C$-ring.

Let $F$ be a finitely generated free $R$-module and $K$ a closed submodule of $F$. Since every closed submodule is neat, $F / K$ is neat-flat by Lemma 2.1. Then $F / K$ is projective by (1), and so $K$ is a direct summand of $F$.

(2) $\Rightarrow(1)$ Let $M$ be a finitely generated neat-flat $R$-module. Then there is an exact sequence $0 \rightarrow \operatorname{Ker}(f) \hookrightarrow F \rightarrow M \rightarrow 0$ with $F$ finitely generated free $R$-module. By Lemma $2.1 \operatorname{Ker}(f)$ is a neat submodule of $F$. Since $R$ is a $C$-ring, $\operatorname{Ker}(f)$ is a closed submodule of $F$ by [11, Theorem 5]. Then $0 \rightarrow \operatorname{Ker}(f) \hookrightarrow F \rightarrow$ $M \rightarrow 0$ is a split exact sequence. Hence $M$ is projective.

Following the proof of Theorem 2.11, we obtain the following corollary.

Corollary 2.12. Every cyclic neat-flat $R$-module is projective if and only if $R$ is right $C S$ and right $C$-ring.

A module $N$ is called semiartinian if every nonzero homomorphic image of $N$ contains a simple module.

Remark 2.13. Let $M$ be an $R$-module. Then the socle series $\left\{S_{\alpha}\right\}$ of $M$ is defined as $S_{1}=\operatorname{Soc}(M), S_{\alpha} / S_{\alpha-1}=\operatorname{Soc}\left(M / S_{\alpha-1}\right)$, and for a limit ordinal $\alpha, S_{\alpha}=\cup_{\beta<\alpha} S_{\beta}$. Put $S=\cup\left\{S_{\alpha}\right\}$. Then, by construction $M / S$ has zero socle. $M$ is semiartinian if and only if $S=M$ (see, for example, [8]).

From the proof of Theorem 2.10, we see that the condition that every free $R$-module is extending implies $R$ is a right $C$-ring. In the following example, we show 
that, if every finitely generated free $R$-module is extending, then $R$ need not be a right $C$-ring. Hence the right $C$-ring condition in 2.11 is not superfluous.

Example 2.14. Let $R$ be the ring of all linear transformations (written on the left) of an infinite dimensional vector space over a division ring. Then $R$ is prime, regular, right self-injective and $\operatorname{Soc}\left(R_{R}\right) \neq 0$ by [13, Theorem 9.12]. As $R$ is a prime ring, $\operatorname{Soc}\left(R_{R}\right)$ is an essential ideal of $R_{R}$. Let $S$ be as in Remark 2.13 , for $M=R$. Then $S \neq R$, by [5, Lemma 1(2)]. Since $R / S$ has zero socle, $S$ is a neat submodule of $R_{R}$. On the other hand, $S$ is not a closed submodule of $R$, otherwise $S$ would be a direct summand of $R$ because $R$ is right self injective (i.e., extending). Therefore, $R$ is not a right $C$-ring. Also, as $R$ is right self injective $R^{n}$ is injective, and so extending for every $n \geq 1$.

\section{N-RINGS}

A commutative domain $R$ is called an $N$-domain if every maximal ideal of $R$ is finitely generated. These domains are characterized as those domains $R$, over which coneat submodules and neat submodules coincide (see, [10]). A ring $R$ is called a right $N$-ring if every maximal right ideal of $R$ is finitely generated.

Remark 3.1. An $R$-module $M$ is said to be FP-injective or absolutely pure if it is pure in every extension, i.e., $\operatorname{Ext}^{1}(N, M)=0$ for each finitely presented $R$-module $N$. If $R$ is a right $N$-ring, then it is easy to see that every pure submodule is neat. So that, in this case, any flat (resp. FP-injective) module is neat-flat (resp. m-injective). An $R$-module $M$ is said to be pure-injective if $M$ is injective relative to all pure exact sequences. The character module $M^{+}$of an $R$-module $M$ is pure injective left $R$ module, and every $R$-module $M$ is a pure submodule of the pure injective $R$-module $M^{++}$(see [9, Proposition 5.3.7]).

The following result will be used in the sequel.

Theorem 3.2 ([3, Theorem 1]). The following statements are equivalent:

(1) $R$ is a right coherent ring;

(2) $M_{R}$ is FP-injective if and only if $M^{+}$is a flat module;

(3) $M_{R}$ is FP-injective if and only if $M^{++}$is an injective right $R$-module;

(4) ${ }_{R} M$ is flat if and only if $M^{++}$is a flat left $R$-module.

Definition 3.3. An $R$-module $M$ is called max-flat if $\operatorname{Tor}_{R}^{1}(M, R / I)=0$ for every maximal left ideal $I$ of $R$ (see [26]).

Note that an $R$-module $M$ is max-flat if and only if $M^{+}$is $m$-injective by the standard isomorphism $\operatorname{Ext}^{1}\left(S, M^{+}\right) \cong \operatorname{Tor}_{1}(M, S)^{+}$, for all simple left $R$-module $S$.

Using the similar arguments of [26, Theorem 4.5], one can prove the following lemma. The proof is omitted.

Lemma 3.4. Let $R$ be a right $N$-ring. The following statements hold:

(1) An R-module $M$ is m-injective if and only if $M^{+}$is max-flat; 
(2) An R-module $M$ is m-injective if and only if $M^{++}$is $m$-injective;

(3) An R-module $M$ is a max-flat left $R$-module if and only if $M^{++}$is a max-flat left $R$-module.

Proposition 3.5. Assume that every neat-flat R-module is flat. Then the following statements hold:

(1) Every m-injective R-module is FP-injective;

(2) For every left R-module $M, M$ is max-flat if and only if $M$ is flat.

Proof. (1) Let $M$ be an $m$-injective $R$-module. By [6, Theorem 3], $\operatorname{Soc}(E(M) / M)=$ 0 , and so $E(M) / M$ is a neat-flat $R$-module. Then $E(M) / M$ is flat by our hypothesis. Hence $M$ is a pure submodule of $E(M)$, and so $M$ is an FP-injective module.

(2) Assume $M$ is a max-flat left $R$-module. Then $M^{+}$is $m$-injective, and so it is FP-injective by (1). But $M^{+}$pure injective by [9, Proposition 5.3.7], so $M^{+}$is injective. Then $M$ is flat by [21, Theorem 3.52]. The converse statement is clear.

Proposition 3.6. Let $R$ be a ring. Consider the following statements:

(1) $R$ is a right $N$-ring and every neat-flat $R$-module is flat;

(2) An R-module $M$ is m-injective if and only if $M^{+}$is flat;

(3) An $R$-module $M$ is m-injective if and only if $M$ is FP-injective, and $R$ is right coherent.

Then $(1) \Rightarrow(2) \Leftrightarrow(3)$.

Proof. $\quad(1) \Rightarrow(3)$ By Proposition 3.5(1), every $m$-injective $R$-module is FP-injective. On the other hand, every FP-injective $R$-module is $m$-injective since every simple $R$ module is finitely presented by (1). Then, for every $R$-module $M, M$ is FP-injective if and only if $M$ is $m$-injective, if and only if $M^{+}$is max-flat by Theorem 3.4(2), if and only if $M^{+}$is a flat module by Proposition 3.5(2). Hence $R$ is a right coherent ring by [3, Theorem 1]. This proves (3).

(2) $\Rightarrow$ (3) Let $M$ be a left $R$-module. We claim that, $M$ is a flat $R$-module if and only if $M^{++}$is a flat module. If $M$ is flat, then $M^{+}$is injective by [21, Theorem 3.52], and so $M^{++}$is flat left $R$-module by (2). Conversely, if $M^{++}$is a flat module, then $M$ is flat since $M$ is a pure submodule of $M^{++}$by [9, Proof of Proposition 5.3.9.], and flat modules are closed under pure submodules (see, [16, Corollary 4.86]). So $R$ is a right coherent ring by Theorem 3.2. The last part of (3) follows by (2) and Theorem 3.2 again.

$$
\text { (3) } \Rightarrow(2) \text { By Theorem 3.2. }
$$

Proposition 3.7. A finite direct product of left $C$-rings is also a left $C$-ring.

Proof. Assume $R$ is a finite direct product of the left $C$-rings $R_{1}, R_{2} \ldots R_{n}$. We will show that $\operatorname{Soc}(R / I) \neq 0$ for each essential left ideal $I$ of $R$. By assumption, $I=I_{1} \times I_{2} \times \cdots \times I_{n}$, where $I_{i} \leq R_{i}$ for $i=1,2, \ldots, n$. Since $I$ is essential in $R$, $I_{i}$ is essential in $R_{i}$ for $i=1,2, \ldots, n$. Then $\operatorname{Soc}\left(R_{i} / I_{i}\right) \neq 0$ for $i=1,2, \ldots, n$. $\operatorname{Soc}(R / I) \cong \prod_{i}^{n} \operatorname{Soc}\left(R_{i} / I_{i}\right) \neq 0$, as desired. 
Set $S a(M):=\Sigma_{M_{i} \in \Lambda} M_{i}$, where $\Lambda$ is the class of all semiartinian submodules $M_{i}$ of $M$. Then $M / S a(M)$ is neat-flat for each $R$-module $M$, because $\operatorname{Soc}(M / S a(M))=0$ by [15, pp. 238].

Note that (1) two-sided hereditary Noetherian rings are $C$-ring by [4, 10.15(3)], and (2) noetherian semiartinian rings are artinian by [23, Proposition 3.1].

Remark 3.8. Let $R$ be a ring and $e$ be a central idempotent in $R$. Then for a right $R$-module $M$ one has, $M=M e \oplus M(1-e)$. It can be easily verified that, $M$ is a neatflat (flat) $R$-module if and only if $M e$ is a neat-flat (flat) $e R$-module and $M(1-e)$ is a neat-flat (flat) $(1-e) R$-module.

Theorem 3.9. Let $R$ be a commutative Noetherian ring. The following statements are equivalent:

(1) Every neat-flat module is flat;

(2) Every absolutely coneat module is FP-injective;

(3) $R \cong A \times B$, wherein $A$ is $Q F$-ring and $B$ is hereditary.

Proof. (1) $\Leftrightarrow$ (2) By [2, Lemma 4.4].

$(1) \Rightarrow(3)$ By the assumption, $R / S a(R)$ is projective and $S a(R)$ is direct summand of $R$, i.e. $R \cong A \times B$, where $A=\operatorname{Sa}(R)$ is $\operatorname{artinian}$, and $\operatorname{Soc}(B)=0$ as $\operatorname{Soc}(R) \leq \operatorname{Sa}(R)$. By Remark 3.8, we can assume $R$ is artinian or $\operatorname{Soc}(R)=0$. In the former case, every neat-flat module is projective by the assumption, and hence $R$ is a $Q F$-ring by Theorem 2.10 and [19, Theorem 4.4]. In the later case, let $I$ be an ideal of $R$. Since $\operatorname{Soc}(R)=0$, we have $\operatorname{Soc}(I)=0$. Then, $I$ is flat by (1) and Corollary 2.5. But $R$ is Noetherian, and so $I$ is finitely generated. Therefore, $I$ is projective, and so $R$ is hereditary.

(3) $\Rightarrow$ (1) Assume that $R \cong A \times B$, wherein $A$ is $Q F$-ring and $B$ is hereditary. Let $M$ be a neat-flat $R$-module. Since $M=M A \oplus M B, M A$ is a neat-flat $A$-module and $M B$ is a neat-flat $B$-module, by Remark 3.8. Then $M A$ is a projective $A$-module by Theorem 2.10, and $M B$ is a flat $B$-module by Corollary 2.8 and [12, Proposition 2.3]. Therefore, $M$ is a flat $R$-module.

Recall that an $R$-module $M$ is said to be weakly-injective if $M$ is coclosed in every extension. $M$ is weakly-injective if and only if $M$ is coclosed in its injective hull (see [27]). Clearly, weakly-injective modules are absolutely coneat.

Theorem 3.10. Let $R$ be a commutative noetherian ring. The following statements are equivalent:

(1) Every weakly-flat module is weakly-injective;

(2) Every weakly-injective module is weakly-flat;

(3) Every neat-flat module is absolutely coneat;

(4) Every absolutely coneat module is neat-flat;

(5) Every neat-flat module is weakly-injective;

(6) Every absolutely coneat module is weakly-flat;

(7) $R \cong A \times B$, wherein $A$ is $Q F$-ring and $B$ is artinian with $J^{2}(B)=0$. 
Proof. $\quad(1) \Leftrightarrow(2) \Leftrightarrow(7)$ By [28, Satz 3.8].

$(5) \Rightarrow(3)$ and $(6) \Rightarrow(4)$ are clear.

(3) $\Rightarrow(4)$ Let $M$ be an absolutely coneat $R$-module. Then $M^{+}$is neat-flat by [2, Proposition 4.3]. By (3), $M^{+}$is absolutely coneat. Again by [2, Proposition 4.3], $M^{++}$is neat-flat. Since $M$ is a pure submodule of $M^{++}, M$ is neat-flat by Proposition 2.6.

(4) $\Rightarrow(3)$ Let $M$ be a neat-flat $R$-module. Then $M^{+}$is absolutely coneat by [2, Proposition 4.3]. By (4), $M^{+}$is neat-flat. Again by [2, Proposition 4.3], $M^{++}$is absolutely coneat. Since $M$ is a pure submodule of $M^{++}, M$ is absolutely coneat by [2, Proposition 3.6].

(7) $\Rightarrow(5)$ A finite direct product of $C$-rings is also a left $C$-ring by Proposition 3.7 , and so $R$ is a $C$-ring. Then neat-flat $R$-modules are weakly-flat and, by [28, Satz 3.8], neat-flat $R$-modules are weakly-injective.

(3) $\Rightarrow$ (7) First we shall prove that, every finitely generated weakly-flat $R$ module is weakly-injective. Let $N$ be a finitely generated weakly-flat $R$-module and $N \leq M$ any extension of $N$. Then $N$ is neat-flat, and absolutely coneat by (3). Then $N I=N \cap M I$ for each maximal ideal $I$ of $R$ by [10]. Since $N$ is finitely generated, it is coatomic (i.e., every submodule $U \lesseqgtr N$ lies in a maximal submodule of $N$ ). Hence $N$ is coclosed in $M$ by [27, Lemma A.3(b)]. Then $N$ is weakly-injective.

The rest of the proof follows as in proof of $\left(i^{\prime} \Rightarrow i i i\right)$ of Satz 3.8 in [28].

$(4) \Rightarrow(6)$ By the equivalence of (4) $\Leftrightarrow(7), R \cong A \times B$, wherein $A$ is a $Q F$-ring and $B$ is artinian with $J^{2}(R)=0$. Now, $R$ is a $C$-ring by Proposition 3.7. Then neatflat $R$-modules are weakly-flat. Therefore, the claim follows by (4).

\section{LOCALIZATION OF NEAT-FLAT MODULES}

In this section, we shall consider localization of neat exact sequences and neatflat modules on commutative $N$-rings.

For an $R$-module $M$ and a prime ideal $P$ of a commutative ring $R$, as usual, $M_{P}$ will be denote the localization of $M$ at $P$. The elements of $M_{P}$ are of the form $\frac{m}{s}$, where $m \in M$ and $s \in R \backslash P . M_{P}$ turns out to be an $R_{P}$-module with multiplication $\frac{r}{s} \cdot \frac{m}{s^{\prime}}=\frac{r m}{s s^{\prime}}$, where $\frac{r}{s} \in R_{P}, \frac{m}{s^{\prime}} \in M_{P}$.

A submodule $A$ of $B$ is neat in $B$ if and only if the following hold: if for $b \in B$ and for a maximal ideal $P$, we have $P b \leq A$, then there is an element $a \in A$ such that $P(b-a)=0$, (see [10, Lemma 2.1]).

We can also rephrase the definition of neat submodule in terms of systems of equations to make the resemblance to purity more transparent: if the maximal ideal $P$ is generated by the elements $r_{i}(i \in I)$, then we consider the system of equations

$$
r_{i} x=a_{i} \in A,(i \in I)
$$

with the single unknown $x$ and constants in $A$.

Lemma 4.1 ([10, Lemma 2.2]). $A$ is neat in $B$ if and only if such systems are solvable in $A$, whenever they are solvable in $B$.

Let $R$ be a commutative ring and $M$ a finitely presented $R$-module. It is well known that $M$ is projective if and only if $M_{P}$ is a free $R_{P}$-module for each prime ideal $P$ of $R$, if and only if $M_{P}$ is a free $R_{P}$-module for each maximal ideal $P$ of $R$. 
Lemma 4.2. Let $R$ be a commutative $N$-ring. Then, a short exact sequence $0 \rightarrow A \rightarrow$ $B \rightarrow C \rightarrow 0$ is neat exact if and only if $0 \rightarrow A_{P} \rightarrow B_{P} \rightarrow C_{P} \rightarrow 0$ is neat exact for each maximal ideal $P$ of $R$.

Proof. $(\Rightarrow)$ Assume that $0 \rightarrow A \stackrel{f}{\rightarrow} B \rightarrow C \rightarrow 0$ is a neat exact sequence of $R$ modules and $P$ is a maximal ideal of $R$. We show that the exact sequence

$$
0 \rightarrow A_{P} \stackrel{f_{P}}{\rightarrow} B_{P} \rightarrow C_{P} \rightarrow 0
$$

is neat exact of $R_{P}$-modules. Assume that $I$ is an index set, and

$$
\frac{r_{i}}{s_{i}} x=\frac{f\left(a_{i}\right)}{s_{i}^{\prime}} \in f_{P}\left(A_{P}\right), \quad r_{i} \in R_{P}, s_{i}, s_{i}^{\prime} \in R \backslash P, a_{i} \in A, i \in I
$$

is a system of equations which is solvable in $B_{P}$, i.e., $\frac{r_{i}}{s_{i}} \frac{b}{l}=\frac{f\left(a_{i}\right)}{s_{i}^{\prime}}$ for some $b \in B$, $l \in R \backslash P$. Thus for each $i \in I$, there exists an element $t_{i} \in R \backslash P$ such that $t_{i} r_{i} s_{i}^{\prime} b=$ $t_{i} s_{i} l f\left(a_{i}\right) \in f(A)$. Now, consider the system of equations $t_{i} r_{i} s_{i}^{\prime} x=t_{i} s_{i} l f\left(a_{i}\right) \in f(A)$ which is solvable in $B$. Since $f(A)$ is a neat submodule of $B$, by Lemma 4.1, there exists an $f(a) \in f(A)$ such that $t_{i} r_{i} s_{i}^{\prime} f(a)=t_{i} s_{i} l f\left(a_{i}\right)$ for each $i \in I$. Thus $\frac{r_{i}}{s_{i}} \frac{f(a)}{l}=\frac{f\left(a_{i}\right)}{s_{i}^{\prime}}$, i.e., the system of equations $\frac{r_{i}}{s_{i}} x=\frac{f\left(a_{i}\right)}{s_{j}^{\prime}}$ is solvable in $f_{P}\left(A_{P}\right)$. Therefore, $f_{P}\left(A_{P}\right)$ is a neat submodule of $B_{P}$ by Lemma 4.1 .

$(\Leftarrow)$ Assume that $0 \rightarrow A \rightarrow B \rightarrow C \rightarrow 0$ is not a neat-exact sequence of $R$ modules but $0 \rightarrow A_{P} \rightarrow B_{P} \rightarrow C_{P} \rightarrow 0$ is neat exact for each maximal ideal $P$ of $R$. Then there is a simple $R$-module $S=R / P$ where $P$ is maximal ideal of $R$ such that $\operatorname{Hom}(S, B) \rightarrow \operatorname{Hom}(S, C)$ is not surjective. By the hypothesis, the natural homomorphism

$$
\operatorname{Hom}_{R_{P}}\left(S_{P}, B_{P}\right) \rightarrow \operatorname{Hom}_{R_{P}}\left(S_{P}, C_{P}\right)
$$

is an epimorphism. Since $S$ is finitely presented, we have the commutative diagram

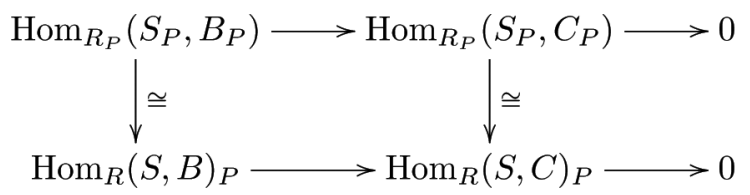

by [21, Lemma 4.87]. Since the $(*)$ row is exact, the $(* *)$ row is also exact.

Note that for a maximal ideal $Q \neq P, \quad S_{Q}=R_{Q} \otimes_{R} S=0$. Therefore, $\operatorname{Hom}_{R}(S, B)_{Q}=\operatorname{Hom}_{R}(S, C)_{Q}=0$. Then $\operatorname{Hom}_{R}(S, B)_{P} \rightarrow \operatorname{Hom}_{R}(S, C)_{P} \quad$ is an epimorphism for every maximal ideal $P$. Thus, by [21, Lemma 4.90], $\operatorname{Hom}_{R}(S, B) \rightarrow$ $\operatorname{Hom}_{R}(S, C)$ is an epimorphism. This contradict with our assumption, and hence $0 \rightarrow A \rightarrow B \rightarrow C \rightarrow 0$ is a neat exact sequence of $R$-modules.

Corollary 4.3. Let $R$ be a commutative $N$-ring. A module $M$ is a neat-flat $R$-module if and only if, for all maximal ideals $P$ of $R, M_{P}$ is a neat-flat $R_{P}$-module. 


\section{ACKNOWLEDGMENTS}

Some part of this article was written while the second author was visiting Padova University, Italy. He would like to thank the members of the Department of Mathematics of Padova University for their hospitality. The authors are grateful to the referee for carefully reading the paper and valuable comments that improved presentation of the paper.

\section{FUNDING}

The second author wishes to thank the Scientific and Technical Research Council of Turkey (TÜBİTAK) for their financial support.

\section{REFERENCES}

[1] Anderson, F. W., Fuller, K. R. (1992). Rings and Categories of Modules. New-York: Springer.

[2] Büyükaşık, E., Durğun, Y. (2015). Absolutely s-pure modules and Neat-flat modules. Comm. Algebra 43(2):384-399.

[3] Cheatham, T. J., Stone, D. R. (1981). Flat and projective character modules. Proc. Amer. Math. Soc. 81(2):175-177.

[4] Clark, J., Lomp, C., Vanaja, N., Wisbauer, R. (2006). Lifting Modules. Basel: Birkhäuser Verlag.

[5] Clark, J., Smith, P. F. (1996). On semi-Artinian modules and injectivity conditions. Proc. Edinburgh Math. Soc. (2) 39(2):263-270.

[6] Crivei, S. (1998). $m$-injective modules. Mathematica 40(63)(1):71-78.

[7] Crivei, S. (2014). Neat and coneat submodules of modules over commutative rings. Bull. Aust. Math. Soc. 89(2):343-352.

[8] Dung, N. V., Huynh, D. V., Smith, P. F., Wisbauer, R. (1994). Extending Modules. Vol. 313, Longman Scientific \& Technical, Harlow.

[9] Enochs, E. E., Jenda, O. M. G. (2000). Relative Homological Algebra Vol. 30, Walter de Gruyter \& Co., Berlin.

[10] Fuchs, L. (2012). Neat submodules over integral domains. Period. Math. Hungar. 64(2):131-143.

[11] Generalov, A. I. (1978). Weak and $\omega$-high purities in the category of modules. Mat. Sb. (N.S.) 105(147)(3):389-402, 463.

[12] Goodearl, K. R. (1972). Singular Torsion and the Splitting Properties. American Mathematical Society, Providence, R. I.

[13] Goodearl, K. R. (1979). von Neumann regular rings. Monographs and Studies in Mathematics, vol. 4, Pitman (Advanced Publishing Program), Boston, Mass.

[14] Honda, K. (1956). Realism in the theory of abelian groups. I. Comment. Math. Univ. St. Paul. 5:37-75.

[15] Kasch, F. (1982). Modules and Rings. London Mathematical Society Monographs, vol. 17, Academic Press, Inc., London-New York.

[16] Lam, T. Y. (1999). Lectures on Modules and Rings. Graduate Texts in Mathematics, vol. 189, New York: Springer-Verlag.

[17] Mao, L. (2007). When does every simple module have a projective envelope? Comm. Algebra 35(5):1505-1516.

[18] Mišina, A. P., Skornjakov, L. A. (1976). Abelian Groups and Modules. Algebra, Logic and Applications, Amer. Math. Soc. Transl. Ser. 2, vol. 107 Providence, RI. 
[19] Oshiro, K. (1984). Lifting modules, extending modules and their applications to QF-rings. Hokkaido Math. J. 13(3):310-338.

[20] Renault, G. (1964). Étude de certains anneaux A liés aux sous-modules compléments dun a-module. C. R. Acad. Sci. Paris 259:4203-4205.

[21] Rotman, J. (1979). An Introduction to Homological Algebra. New York: Academic Press.

[22] Sandomierski, F. L. (1968). Nonsingular rings. Proc. A23mer. Math. Soc. 19:225-230.

[23] Shock, R. C. (1974). Dual generalizations of the Artinian and Noetherian conditions. Pacific J. Math. 54(2):227-235.

[24] Smith, P. F. (1981). Injective modules and prime ideals. Comm. Algebra 9(9):989-999.

[25] Wang, M. Y., Zhao, G. (2005). On maximal injectivity. Acta Math. Sin. (Engl. Ser.) 21(6): 1451-1458.

[26] Xiang, Y. (2010). Max-injective, max-flat modules and max-coherent rings. Bull. Korean Math. Soc. 47(3):611-622.

[27] Zöschinger, H. (2006). Schwach-injektive moduln. Period. Math. Hungar. 52(2):105-128.

[28] Zöschinger, H. (2013). Schwach-Flache moduln. Comm. Algebra 41(12):4393-4407. 